RAIRO Operations Research

RAIRO Oper. Res. 37 (2003) 291-309

DOI: $10.1051 /$ ro:2004002

\title{
ANALYSE DE SENSIBILITÉ POUR LES PROBLÈMES LINÉAIRES EN VARIABLES 0-1
}

\author{
Babacar Thiongane $^{1}$, Anass Nagih ${ }^{1}$ et Gérad Plateau ${ }^{1}$
}

\begin{abstract}
This paper is a state of the art on sensitivity analysis for 0-1 linear programming problems. Several aspects are considered: history and forms of sensitivity analysis, application examples, complexity, optimality conditions, existing algorithms and approaches.
\end{abstract}

Résumé. Cet article est un travail de synthèse autour de l'analyse de sensibilité pour les problèmes linéaires en variables 0-1. De nombreux aspects sont ainsi abordés : historique et formes d'analyse de sensibilité, exemples d'application, complexité, conditions d'optimalité, algorithmes et approches. Nous dressons par ailleurs quelques perspectives de recherche actuelles dans ce domaine.

Mots Clés. Analyse de sensibilité, réoptimisation, rayon de stabilité, problèmes linéaires en $0-1$.

\section{INTRODUCTION}

Étant donné un problème d'optimisation combinatoire $(P)$, l'analyse postoptimale, l'analyse de sensibilité, ou l'analyse de stabilité d'une solution de $(P)$, sont autant de termes pour désigner l'étude de la stabilité de cette solution en fonction d'une évolution des données du problème, ou bien l'étude des possibilités de résolution efficace d'un problème $\left(P^{\prime}\right)$ obtenu à partir de $(P)$ en modifiant une partie des données (réoptimisation).

1 LIPN, UMR CNRS 7030, Université de Paris, 99 avenue Jean-Baptiste Clément, 93430 Villetaneuse, France; e-mail : babacar.thiongane@lipn.univ-paris13.fr 
Cette problématique traitée efficacement de longue date pour les problèmes linéaires en variables continues devient plus difficile lorsque les variables sont entières.

Des synthèses sur l'analyse de sensibilité en optimisation combinatoire ont déjà été effectuées dans $[14,18,22,50]$. Dans [14] les auteurs présentent l'état de l'analyse de sensibilité lorsqu'elle balbutiait dans les années 70 . Un apport important dans $[14,18]$ a surtout porté sur la paramétrisation qui va au-delà de l'analyse de sensibilité, et dont nous ne parlerons pas dans ce papier (pour des travaux sur la paramétrisation pour les problèmes en nombres entiers voir $[4,7,8,14,19,23,24,34,35])$. Pour le cas des problèmes linéaires en nombres entiers une synthèse plus riche a été faite dans [22]. Enfin dans [50] une synthèse plus complète sur l'analyse de sensibilité pour les problèmes d'optimisation combinatoire a été réalisée, avec un accent particulier pour les problèmes polynomiaux.

Nous nous intéressons ici à différents aspects relatifs à l'analyse de sensibilité pour les problèmes linéaires en variables 0-1 en y incluant un volet sur la réoptimisation. Notre synthèse s'appuie sur de nombreux travaux [17], et met davantage l'accent sur les modifications de données relatives à la fonction objectif. La part de travaux publiés sur l'analyse de sensibilité concernant la fonction objectif est par ailleurs plus importante que celle consacrée aux contraintes.

Dans la section 2, nous abordons les différentes formes d'analyse de sensibilité pour les problèmes linéaires en 0 -1. Ensuite un historique relatif à l'analyse de sensibilité sera présenté dans la section 3, puis nous verrons dans la section 4 quelques exemples d'applications.

Dans la section 5 nous abordons la complexité de l'analyse de sensibilité des problèmes d'optimisation combinatoire ce qui nous conduit dans la section 6 à l'étude des conditions d'optimalité, fondements de la plupart des algorithmes existants sur l'analyse de sensibilité.

Nous verrons ainsi dans la section 7 comment à partir de ces conditions il a été possible de construire des algorithmes et des approches pour les différentes formes d'analyse de sensibilité.

Enfin la section 8 mentionne quelques perspectives de recherche actuelles dans le domaine de l'analyse de sensibilité.

\section{LES DIFFÉRENTES FORMES D'ANALYSE DE SENSIBILITÉ}

Soit $c \in \mathbb{R}^{n}$. On considère le problème linéaire défini par :

$$
\begin{array}{lll}
(P) & \max & c x \\
& \text { s.c } & x \in X \subset\{0,1\}^{n} .
\end{array}
$$

Soit $x^{*} \in \Omega(P)$ où $\Omega(P)$ désigne l'ensemble des solutions optimales de $(P)$. On définit alors la région de stabilité $\mathcal{R}$ de la solution $x^{*}$ par

$$
\mathcal{R}=\left\{\left(c^{\prime}, X^{\prime}\right) \mid c^{\prime} \in \mathbb{R}^{n}, X^{\prime} \subset\{0,1\}^{n}, x^{*} \in \Omega\left(P^{\prime}\right)\right\}
$$


où $\left(P^{\prime}\right)$ est le problème suivant

$$
\begin{array}{lll}
\left(P^{\prime}\right) & \max & c^{\prime} x \\
& \text { s.c } & x \in X^{\prime} \subset\{0,1\}^{n} .
\end{array}
$$

$\mathcal{R}$ représente en fait l'ensemble de toutes les variations des données qui laisse la solution $x^{*}$ optimale. En général il est très difficile de décrire l'ensemble $\mathcal{R}$. Le plus souvent on ne cherche à décrire qu'un sous-ensemble comme l'intervalle de sensibilité de $c_{k}, k \in\{1, . ., n\}$ (voir Sect. 2.1), ou bien une boule de stabilité (voir Sect. 2.3). Lorsque $\left(c^{\prime}, X^{\prime}\right) \notin \mathcal{R}$, plutôt que de résoudre $\left(P^{\prime}\right)$ de manière directe, il peut être intéressant d'essayer de le résoudre en exploitant la résolution de $(P)$ afin d'économiser en temps d'exécution; on parle alors de réoptimisation (voir Sect. 2.2).

\subsection{L'INTERVALLE DE SENSIBILITÉ}

Soient $k \in\{1, . ., n\},\left[\underline{c}_{k}, \bar{c}_{k}\right]$ un intervalle de $\mathbb{R}$ contenant $c_{k}$, et $c^{\prime} \in \mathbb{R}^{n}$ tel que $c_{j}^{\prime}=c_{j}$ pour tout $j \in\{1, . ., n\} \backslash\{k\}$ et $c_{k}^{\prime} \in\left[\underline{c}_{k}, \bar{c}_{k}\right]$ avec $c_{k}^{\prime} \neq c_{k}$. Soit

$$
\begin{array}{lll}
\left(P^{\prime}\right) & \max & c^{\prime} x \\
& \text { s.c } & x \in X \subset\{0,1\}^{n} .
\end{array}
$$

On dit que $\left[\underline{c}_{k}, \bar{c}_{k}\right]$ est un intervalle de sensibilité, ou intervalle de tolérance, pour le coefficient $c_{k}$, si pour tout $c_{k}^{\prime}$ dans $\left[\underline{c}_{k}, \bar{c}_{k}\right], x^{*} \in \Omega\left(P^{\prime}\right)$. Étant donné une solution optimale, l'intervalle de sensibilité d'un coefficient est donc un intervalle de valeurs dans lequel cette solution reste optimale. Si pour toute valeur $c_{k}^{\prime} \notin\left[\underline{c}_{k}, \bar{c}_{k}\right]$, $x^{*}$ n'est plus optimale, alors $\left[\underline{c}_{k}, \bar{c}_{k}\right]$ est un intervalle de sensibilité exact pour le coefficient $c_{k}$.

Il est également possible de chercher à déterminer un intervalle de sensibilité pour plusieurs coefficients à la fois. Par ailleurs lorsque l'ensemble $X$ s'exprime par un système de contraintes, l'intervalle de sensibilité peut concerner un coefficient des contraintes ou du second membre des contraintes.

\subsection{LA RÉOPTIMISATION}

Supposons que le problème $(P)$ a été résolu. Soit $\left(P^{\prime}\right)$ le problème défini à partir de $(P)$ en modifiant une partie des données, on suppose en particulier que $\left(P^{\prime}\right)$ est défini à partir de $\left(c^{\prime}, X\right)$ ou de $\left(c, X^{\prime}\right)$, avec $c^{\prime} \in \mathbb{R}^{n}$ et $X^{\prime} \subset\{0,1\}^{n}$. La réoptimisation consiste en l'étude théorique et algorithmique des possibilités de résoudre efficacement $\left(P^{\prime}\right)$ en exploitant la résolution de $(P)$. La réoptimisation sera d'autant plus efficace que la résolution de $(P)$ s'est effectuée dans la perspective de résoudre $\left(P^{\prime}\right)$ efficacement et que des informations pertinentes ont été sauvegardées pour être réexploitées.

\subsection{Les RAyon et Boule de Stabilité}

La définition du rayon et de la boule de stabilité est exclusivement réservée à la fonction objectif $c$ et ne concerne donc pas l'ensemble $X$. On considère une 
distance sur $\mathbb{R}^{n}$, disons la distance de Chebyshev $d$ définie par :

$$
d\left(c, c^{\prime}\right)=\max \left\{\left|c_{j}-c_{j}^{\prime}\right|, j \in\{1, . ., n\}\right\}
$$

où $c$ et $c^{\prime}$ sont des vecteurs de $\mathbb{R}^{n}$.

On dit que

$$
O_{\rho}(c)=\left\{c^{\prime} \in \mathbb{R}^{n} \mid d\left(c, c^{\prime}\right) \leq \rho\right\}
$$

est une boule de stabilité de centre $c \in \mathbb{R}^{n}$ et de rayon $\rho \geq 0$ si pour tout $c^{\prime} \in O_{\rho}(c)$, $\Omega\left(P^{\prime}\right) \subseteq \Omega(P)$, où $\left(P^{\prime}\right)$ est le problème défini par $\left(c^{\prime}, X\right)$. Le rayon de stabilité $\rho(c)$ est alors défini comme le plus grand $\rho \geq 0$ tel que $O_{\rho}(c)$ est une boule de stabilité.

\section{UN PEU D'HISTOIRE...}

De manière générale, l'analyse de sensibilité pour les problèmes d'optimisation combinatoire est apparue peu de temps après les méthodes de résolution exacte de ces problèmes. Pour les problèmes linéaires en $0-1$ auxquels nous nous intéressons ici, le premier algorithme a été proposé au début des années 70. L'analyse de sensibilité pour ces problèmes a donc une trentaine d'années. C'est Roodman qui le premier, s'appuyant sur l'algorithme d'énumération implicite de Balas [1], a présenté en 1972 une première approche pour appréhender le problème de l'analyse de sensibilié dans un contexte spécifique. À partir de là, d'autres auteurs ont proposé d'abord des améliorations de l'algorithme de Roodman ensuite des contributions plus originales sur l'analyse de sensibilité. Tous ces travaux ont porté essentiellement sur "l'intervalle de sensibilité" et la "réoptimisation" qui sont des termes propres à la littérature occidentale. Parallèlement à cela, et à partir du milieu des années 70, apparut un autre concept de l'analyse de sensibilité appelé rayon de stabilité. Ce concept théorique a été introduit pour la première fois par Leontev [28,29]. De très nombreux travaux ont été consacrés à l'étude du rayon de stabilité et les termes "d'analyse de stabilité" sont en réalité propres à la littérature russe. Il est à noter que l'ensemble des travaux de cette littérature est resté pendant longtemps difficilement accessible à la communauté occidentale. C'est dans un article apparu au milieu des années 90 que Sotskov, Leontev et Gordeev [44] font un état de l'art des travaux sur le rayon de stabilité pour de nombreux problèmes d'optimisation combinatoire et présentent une centaine de références bibliographiques essentiellement en russe.

\section{EXEMPLES D'APPLiCATION}

L'intérêt de l'analyse de sensibilité est mis en évidence lorsque dans la modélisation de problèmes réels certaines données de la fonction objectif à optimiser sont encore imprécises au moment de l'étude et qu'on est amené à considérer des estimations de ces données réelles. Dans un problème d'investissement il est rare qu'on puisse connaître précisément le montant du retour sur investissement d'un projet. On est souvent amené à consulter un expert qui va évaluer ce montant avec 
un intervalle d'incertitude. Il peut alors être intéressant de faire le rapport entre cet intervalle d'incertitude et l'intervalle de sensibilité.

Une autre application intervient lorsqu'il existe un environnement dynamique qui influe sur les valeurs des données du problème comme cela est le cas pour le problème de dimensionnement de lots en horizon infini [20]. On considère $T$ périodes consécutives $1, \ldots, T$ et un ensemble de demandes de fabrication d'un produit sur ces périodes. Le coût total de chaque période est calculé en fonction du coût de production et du coût de stockage durant cette période. Le premier est composé d'un coût fixe s'il y a eu production durant la période et d'un coût unitaire de production. En supposant que toutes les données sont connues, le problème de dimensionnement de lots consiste à satisfaire la demande à moindre coût. Plaçons nous dans le contexte où ce problème a été résolu et supposons que le plan de production issu de la résolution est en cours d'exécution; disons qu'on se situe alors à la période $t<T$. Supposons maintenant que les informations sur les données de la période $T+1$ deviennent disponibles. On voudrait alors savoir si le plan de production initial doit être revu ou peut rester inchangé. En d'autres termes il s'agirait d'étudier l'analyse de sensibilité de la planification originelle de production.

Dans un cadre plus théorique l'analyse de sensibilité peut servir à accélérer un schéma de résolution dans lequel la connaissance de l'intervalle de sensibilité d'une solution évite des résolutions inutiles, ou dans lequel la résolution d'un problème peut être accélérée par réoptimisation, comme cela est le cas dans la résolution d'un problème de paramétrisation par une approche par encadrement $[23,35]$.

\section{Complexité}

À l'inverse des problèmes linéaires à variables continues, les problèmes liés à l'analyse de sensibilité sont pour la plupart des problèmes d'optimisation combinatoire NP-difficiles.

Gordeev a montré dans [16] que bien que le problème du plus court chemin soit un problème polynomial, la détermination du rayon de stabilité est NP-difficile.

Lorsque le problème $(P)$ est un problème de maximisation d'une forme linéaire booléenne

$$
\begin{array}{lll}
(P) & \max & c x \\
& \text { s.c } & x \in X \subset\{0,1\}^{n}
\end{array}
$$

où $c \in \mathbb{R}_{+}^{n}$, Van Hoesel et Wagelmans ont montré dans [21] que l'existence d'un algorithme polynomial pour déterminer les intervalles de sensibilité des coefficients $c_{k}$ implique l'existence d'un algorithme polynomial pour résoudre $(P)$. Pour ce faire il faut se placer sous les deux hypothèses suivantes :

$\left(\mathcal{H}_{1}\right)$ pour tout $c^{\prime} \in \mathbb{R}_{+}^{n}$, on peut déterminer en temps polynomial une solution $x^{\prime} \in X$ telle que $x_{j}^{\prime} \geq x_{j}, j=1, . ., n$, pour tout $x \in X$

$\left(\mathcal{H}_{2}\right)$ pour tout $c^{\prime} \in \mathbb{R}_{+}^{n}$, on peut déterminer en temps polynomial l'intervalle de sensibilité associé à $c_{k}^{\prime}$ pour tout $k=1, . ., n$.

Pour montrer le résultat on procède en deux grandes phases. Soit $x^{\prime}$ vérifiant la propriété définie dans l'hypothèse $\left(\mathcal{H}_{1}\right)$. On construit une instance $\left(P^{\prime \prime}\right)$ définie à 
partir de $\left(c^{\prime \prime}, X\right)$ et telle que $x^{\prime} \in \Omega\left(P^{\prime \prime}\right)$. Pour ce faire on prend $c^{\prime \prime}$ tel que $c_{j}^{\prime \prime}=c_{j}$ si $x_{j}^{\prime}=1$ et $c_{j}^{\prime \prime}=M$ si $x_{j}^{\prime}=0$, avec $M=\min \left\{c_{j}-1, j=1, . ., n\right\}$. La deuxième phase consiste à construire une suite d'instances à partir de $\left(P^{\prime \prime}\right)$, jusqu'à obtenir l'instance $(P)$. Chaque instance ne se différencie de la précédente que par un coefficient d'indice $j$. Le but est de faire passer un coefficient égal à $M$ à la valeur $c_{j}$. Le nombre d'instances engendrées sera donc au plus égal à $n$. L'hypothèse $\left(\mathcal{H}_{2}\right)$ permet alors de déterminer en temps polynomial une solution optimale de l'instance courante. Par suite, une solution optimale de $(P)$ sera obtenue en temps polynomial. Ainsi, à moins que $\mathcal{P}=\mathcal{N} \mathcal{P}$, la détermination de l'intervalle de sensibilité des coefficients d'un problème linéaire en 0-1 est NP-difficile.

\section{Conditions D’optimalité}

La plupart des algorithmes et approches existants pour l'analyse de sensibilité reposent sur des conditions d'optimalité [32]. En programmation linéaire il existe une condition nécessaire et suffisante d'optimalité qui est basée sur le signe des coûts réduits des variables hors-base. Dans ce cas, l'analyse de sensibilité découle de l'intervalle obtenu à partir de cette condition d'optimalité. Pour bon nombre de problèmes d'optimisation combinatoire, il n'existe pas de conditions nécessaires et suffisantes d'optimalité. Souvent c'est en exprimant finement, pour des problèmes spécifiques, des conditions suffisantes d'optimalité qu'on arrive à construire un algorithme pour l'analyse de sensibilité. Cinq types de conditions sont fréquemment rencontrées dans la littérature.

\section{(i) Conditions triviales}

Les conditions triviales d'optimalité peuvent se formuler comme suit :

$$
x^{*} \in \Omega(P) \Longleftrightarrow x^{*} \in X \text { et } c x^{*} \geq c x \forall x \in X
$$

De manière générale tous les algorithmes pour l'analyse de sensibilité s'appuyent entre autres sur ces conditions comme nous le verrons dans la section 7.1.1.

\section{(ii) Conditions basées sur les K meilleures solutions}

On suppose déterminées les $\mathrm{K}$ meilleures solutions $x^{1}, \ldots, x^{K}$ du problème

$$
\begin{aligned}
& (P) \quad \max c x \\
& \text { s.c } \quad x \in X \subset\{0,1\}^{n}
\end{aligned}
$$

avec plus précisément, $c x^{1} \geq \ldots \geq c x^{K}$ et $x^{1} \in \Omega(P)$. Soient $c^{\prime} \in \mathbb{R}^{n}, c^{\prime} \neq c$ et $\left(P^{\prime}\right)$ le problème obtenu en remplaçant $c$ par $c^{\prime}$ dans $(P)$. Piper et Zoltners [37] ont exploité la propriété suivante. Soit $i \in\{1, . ., K\}$ :

$$
\text { si } c^{\prime} x^{i} \geq c^{\prime} x \forall x \in\left\{x^{1}, \ldots, x^{K}\right\} \backslash\left\{x^{i}\right\} \text {, et } c^{\prime} x^{i} \geq z\left(c^{\prime}\right),
$$

où

alors $x^{i} \in \Omega\left(P^{\prime}\right)$

$$
z\left(c^{\prime}\right)=\max \left\{c^{\prime} x \mid c x \leq c x^{K}, x \in X\right\}
$$


En pratique, pour vérifier si $\left\{x^{1}, \ldots, x^{K}\right\}$ contient une solution optimale pour le problème $\left(P^{\prime}\right)$, les auteurs considèrent généralement un majorant de $z\left(c^{\prime}\right)$ en résolvant une relaxation de $\max \left\{c^{\prime} x \mid c x \leq c x^{K}, x \in X\right\}$ (voir Sect. 7.1.2).

(iii) Conditions relevant de méthodes spécifiques

Dans les méthodes d'optimisation, qu'elles soient classiques ou spécifiques, il existe des conditions particulières (suffisantes) qui lorsqu'elles sont vérifiées garantissent l'optimalité de la solution obtenue. La méthode de branch \& bound par exemple est une méthode de résolution qui consiste à explorer l'espace des solutions du problème et à générer des solutions partielles en dirigeant l'exploration vers des sous-espaces prometteurs. Des tests d'élagage (optimalité, réalisabilité) permettent de détecter les solutions partielles non prometteuses. Ces tests constituent des conditions de non optimalité. Nous verrons plus loin comment faire de l'analyse de sensibilité en exploitant les conditions de non optimalité avec une méthode de branch \& bound (voir Sect. 7.1.3).

(iv) Conditions basées sur la programmation linéaire

Il s'agit ici de chercher à décrire l'enveloppe convexe des points entiers pour pouvoir appliquer les résultats de la programmation linéaire sur l'analyse de sensibilité. La description de ce polyèdre est un problème NP-difficile mais seule une description dans le voisinage de la solution optimale est nécessaire (voir Sect. 7.1.4).

\section{(v) Conditions basées sur la dualité}

En général la dualité Lagrangienne ne permet d'obtenir qu'un majorant de la valeur optimale du problème $(P)$. Avec la théorie de la dualité convergente il est possible de construire un problème dual sans saut de dualité. Lorsque le saut de dualité est nul alors une solution optimale de $(P)$ est obtenue. L'analyse de sensibilité qui est basée sur cette condition d'optimalité consiste à déterminer l'intervalle de sensibilité à travers la fonction duale (voir Sect. 7.1.5).

\section{Algorithmes et approches pour l'analyse de Sensibilité}

Nous allons voir ici comment il est possible, en s'appuyant sur les conditions d'optimalité énumérées dans le paragraphe précédent, de construire des algorithmes pour la détermination d'un intervalle de sensibilité (Sect. 7.1) ou pour la résolution d'un problème par réoptimisation (Sect. 7.2).

Dans le cas du rayon de stabilité, l'existence d'une expression analytique fait qu'en général on ne cherche pas un algorithme mais plutôt une approche mathématique pour simplifier cette expression complexe.

\subsection{Détermination D’Un intervalle De Sensibilité}

On résume ici les cas d'utilisation des cinq types de conditions décrites dans la section 6 . 


\subsubsection{Conditions triviales}

Pour le problème du sac à dos unidimensionnel en variables $0-1$, c'est-à-dire pour le cas particulier où

$$
X=\left\{x \in\{0,1\}^{n} \mid a x \leq b\right\}
$$

avec $b, c_{j}, a_{j} \in \mathbb{R}_{+}$pour tout $j \in\{1, . ., n\}$, Libura [31] a montré que les intervalles de sensibilité des coefficients du premier et du second membre des contraintes se déduisent de la construction de l'ensemble suivant :

$$
\left\{\left(a_{1}, \ldots, a_{n}, b\right) \in \mathbb{R}_{+}^{n+1} \mid a x^{*} \leq b, \sum_{i \in J} a_{i}>b \forall J \in \mathcal{Q}\right\}
$$

où $x^{*} \in \Omega(P)$ et $\mathcal{Q}$ est une famille maximale de sous-ensembles $J \subseteq\{1, . ., n\}$ tels que $\sum_{i \in J} c_{i}>c x^{*}$. Ce résultat est en fait obtenu à partir de la définition de l'optimalité pour ce contexte spécifique. Libura ne donne pas d'algorithme pour le calcul de cet intervalle de sensibilité mais cela permet de voir comment la définition de l'optimalité permet d'obtenir une description plus formelle de l'intervalle.

\subsubsection{Exploitation des $K$ meilleures solutions}

Piper et Zoltners [37] ont proposé une méthode pour déterminer un intervalle de sensibilité relativement à $c$ pour les coefficients d'un problème $(P)$ de maximisation défini par $(c, X)$. Supposons que $(P)$ est un problème linéaire en $0-1$ et que $c_{k} \in[0,+\infty[, k \in\{1, . ., n\}$. Alors l'algorithme de branch \& bound peut-être modifié pour générer les $\mathrm{K}$ meilleures solutions $\left\{x^{1}, . ., x^{K}\right\}$ de $(P)$. Pour ce faire Piper et Zoltners considère un paramètre dynamique $\delta>0$. Ils proposent d'effectuer le test d'élagage du branch \& bound non plus en fonction de la valeur $c x^{1}$ de la meilleure solution connue mais en fonction de la valeur $c x^{1}-\delta$. Lorsque qu'une K-ième meilleure solution $x^{K}$ a été trouvée pour la première fois au cours de l'algorithme alors $c x^{1}-\delta$ est réactualisé à $c x^{K}$. Ainsi on récupère les $\mathrm{K}$ meilleures solutions qui sont à une distance $\delta$ de l'optimum (on suppose au départ $\delta$ suffisamment grand pour pouvoir trouver les $K$ meilleurs solutions sinon on en trouve moins).

Soit $\left(P^{\prime}\right)$ le problème défini à partir de $\left(c^{\prime}, X\right)$ où $c^{\prime}$ ne se différencie de $c$ que par rapport à l'indice $k$. L'analyse de sensibilité considérée par Pipers et Zoltners consiste à déterminer l'intervalle de sensibilité de $c_{k}$ garantissant la propriété qu'une solution optimale de $\left(P^{\prime}\right)$ se trouve dans l'ensemble de solutions $\left\{x^{1}, . ., x^{K}\right\}$. Piper et Zoltners montrent alors que si une variable est fixée à 1 dans une solution de cet ensemble et à 0 dans une autre solution de ce même ensemble, alors l'intervalle de sensibilité du coefficient de cette variable est $[0,+\infty[$. Si en revanche cette variable est fixée à 1 (resp. 0) dans toute solution de l'ensemble $\left\{x^{1}, . ., x^{K}\right\}$, alors l'intervalle de sensibilité correspondant est $\left[c_{k}-\delta,+\infty\left[\left(\operatorname{resp} .\left[0, c_{k}+\delta\right]\right)\right.\right.$ avec $\delta=c x^{1}-c x^{K}$. 


\subsubsection{Conditions relevant de méthodes spécifiques}

Historiquement les premiers travaux sur l'analyse de sensibilité ont été basés sur les conditions induites par les méthodes d'exploration arborescente [36-39]. À l'issue d'une méthode d'énumération implicite ou de branch \& bound, on obtient un ensemble de solutions complètes (réalisables) qui contient la solution optimale, et un ensemble de solutions partielles (par arbitrage d'un sous-ensemble de variables) qui ont été élaguées par l'optimalité lors de l'énumération.

Supposons toujours que le problème $(P)$ défini par $(c, X)$ dans lequel les données $c_{j}, j \in\{1, . ., n\}$ sont positives uniquement pour simplifier les explications. Après résolution de $(P)$, on obtient donc une arborescence associée à deux grands ensembles : Ec qui contient les solutions complètes et Ep qui regroupe les solutions partielles.

Le principe de l'analyse de sensibilité consiste ici à exploiter les informations liées à cette arborescence pour déterminer l'intervalle de sensibilité d'une donnée de $(P)$.

Pour un coefficient $c_{k}$ donné, on cherche un intervalle de valeurs $\left[\underline{c}_{k}, \bar{c}_{k}\right)$ tel que la solution optimale $x^{*}$ de $(P)$ est aussi solution optimale du problème $\left(P^{\prime}\right)$ obtenu à partir de $(P)$ en ne modifiant que la valeur de $c_{k}$ dans $\left[\underline{c}_{k}, \bar{c}_{k}\right)$.

$$
\begin{array}{lll}
\left(P^{\prime}\right) & \max & c^{\prime} x \\
& \text { s.c } & x \in X \subset\{0,1\}^{n}
\end{array}
$$

où $c_{j}^{\prime}=c_{j}, j \in\{1, . ., n\} \backslash\{k\}$.

Pour déterminer cet intervalle de sensibilité on distingue deux cas : $x_{k}^{*}=0$ (cas 1$)$ et $x_{k}^{*}=1$ (cas 2). Dans chacun des cas on obtient une borne évidente de l'intervalle de sensibilité. En effet les données étant supposées positives, dans le premier cas $\underline{c}_{k}=0$, dans le second $\bar{c}_{k}=+\infty$.

Le calcul de la deuxième borne de l'intervalle de sensibilité repose en fait sur une idée simple. Supposons que $x_{k}^{*}=\epsilon, \epsilon \in\{0,1\}$. Alors la solution $x^{*}$ reste optimale pour $\left(P^{\prime}\right)$ tant qu'elle est meilleure que la solution obtenue en fixant $x_{k}$ à la valeur $1-\epsilon$, ce qui se traduit par

$$
v\left(P^{\prime} \mid x_{k}=1-x_{k}^{*}\right) \leq v\left(P^{\prime} \mid x_{k}=x_{k}^{*}\right)
$$

où $v\left(P^{\prime} \mid x_{k}=1-x_{k}^{*}\right)$ (resp. $\left.v\left(P^{\prime} \mid x_{k}=x_{k}^{*}\right)\right)$ désigne la valeur optimale de $\left(P^{\prime}\right)$ lorsque $x_{k}=1-x_{k}^{*}\left(\operatorname{resp} . x_{k}=x_{k}^{*}\right)$.

$\underline{\operatorname{Cas} 1}\left(x_{k}^{*}=0\right)$

L'inégalité se traduit par :

$$
v\left(P \mid x_{k}=1\right)-c_{k}+c_{k}^{\prime} \leq v(P)(*)
$$

et $\bar{c}_{k}$ est donc la plus grande valeur de $c_{k}^{\prime}$ telle que $(*)$ est vraie, c'est-à-dire $\bar{c}_{k}=c_{k}+v(P)-v\left(P \mid x_{k}=1\right)$. Le calcul de $\bar{c}_{k}$ nécessite donc la résolution de $\left(P \mid x_{k}=1\right)$. 
$\underline{\operatorname{Cas} 2}\left(x_{k}^{*}=1\right)$

L'inégalité devient

$$
v\left(P \mid x_{k}=0\right) \leq v(P)-c_{k}+c_{k}^{\prime}(* *)
$$

et $\underline{c}_{k}$ est la plus petite valeur de $c_{k}^{\prime}$ telle que $(* *)$ est vraie, soit $\underline{c}_{k}=c_{k}+v\left(P \mid x_{k}=\right.$ $0)-v(P)$. Ce qui nécessite la résolution de $\left(P \mid x_{k}=0\right)$.

Il suffit donc d'exploiter l'arborescence obtenue en résolvant $(P)$ pour résoudre ou bien $\left(P \mid x_{k}=1\right)$ ou $\left(P \mid x_{k}=0\right)$ selon le cas. D'où l'algorithme de détermination de l'intervalle de sensibilité $I_{k}$ associé à $c_{k}$ suivant :

1. résoudre $(P)$ par branch \& bound en sauvegardant les solutions partielles Ep et complètes Ec engendrées;

2. soit $\epsilon$ la valeur de $x_{k}^{*}$. Effectuer une deuxième phase d'énumération à partir des solutions partielles de Ep telles que $x_{k}=1-\epsilon$ et celles dans lesquelles $x_{k}$ est libre (dans ce dernier cas fixer $x_{k}$ à $1-\epsilon$ ). En notant : $x^{p}$ : la meilleure solution ainsi obtenue à partir de Ep; $x^{c}$ : la meilleure solution de Ec telle que $x_{k}=1-\epsilon$; $x^{m}$ : la meilleure solution de $x^{p}$ et $x^{c}$, on en déduit que :

(i) si $\epsilon=0$ alors $\bar{c}_{k}=c_{k}+v(P)-c x^{m}$ et $I_{k}=\left[0, \bar{c}_{k}\right]$;

(ii) si $\epsilon=1$ alors $\underline{c}_{k}=c_{k}+c x^{m}-v(P)$ et $I_{k}=\left[\underline{c}_{k},+\infty\right]$.

\subsubsection{Conditions basées sur la programmation linéaire}

Klein et Holm [26] ont étudié l'analyse de sensibilité à partir de ces conditions. Considérons à nouveau le problème $(P)$ dans lequel

$$
X=\left\{x \in\{0,1\}^{n} \mid A x \leq b\right\}
$$

avec $A \in \mathbb{N}^{m n}$ et $b, c \in \mathbb{N}^{m}$. On considère une résolution de $(P)$ par une méthode de coupes. Celle-ci consiste à résoudre initialement le problème $(\bar{P})$ obtenu en relâchant les contraintes d'intégrité de $(P)$. Tant que la solution de $(\bar{P})$ n'est pas binaire, une coupe de Gomory adéquate est insérée dans l'ensemble des contraintes de $(\bar{P})$ et on résout le nouveau problème $(\bar{P})$ obtenu. Notons encore $(\bar{P})$ le dernier problème obtenu par la méthode des coupes.

$$
\begin{array}{ll}
(\bar{P}) \quad \max & c x \\
\text { s.c } & x \in \bar{X}
\end{array}
$$

où $\bar{X}=\left\{x \in[0,1]^{n} \mid \bar{A} x \leq \bar{b}\right\}$. L'analyse de sensibilité découle alors de la non positivité des coûts réduits optimaux des variables hors-base. Soit

$$
c-c_{B} B^{-1} \bar{A} \leq 0
$$

où $c_{B}$ est le vecteur des coûts réduits des variables de base, et $B^{-1}$ est l'inverse de la matrice de base optimale.

Néanmoins cette approche n'offre pas d'aussi bons résultats théoriques sur l'analyse de sensibilité que le permet le cas continu dont il est pourtant analogue dans le principe. En effet l'intervalle de sensibilité obtenu n'est pas exact. Cela est illustré sur cet exemple de problème en nombres entiers proposé par Garfinkel et Nemhauser [13] : 


$$
\begin{array}{ll}
(P) \quad \max & 2 x_{1}+x_{2} \\
\text { s.c } & x_{1}+x_{2} \leq 5 \\
& -x_{1}+x_{2} \leq 0 \\
& 6 x_{1}+2 x_{2} \leq 21 \\
& x_{1}, x_{2} \in \mathbb{N} .
\end{array}
$$

Après résolution par la méthode des coupes on obtient le problème suivant :

$$
\begin{array}{ll}
\left(P^{\prime}\right) \quad \max & 2 x_{1}+x_{2} \\
\text { s.c } & x_{1}+x_{2} \leq 5 \\
& -x_{1}+x_{2} \leq 0 \\
& 6 x_{1}+2 x_{2} \leq 21 \\
& 2 x_{1}+x_{2} \leq 7 \\
& x_{2} \leq 2 \\
& x_{1}+x_{2} \leq 4 \\
& x_{1}, x_{2} \geq 0
\end{array}
$$

où $(1,2)$, et $(3)$ représentent les coupes de Gomory engendrées par le processus.

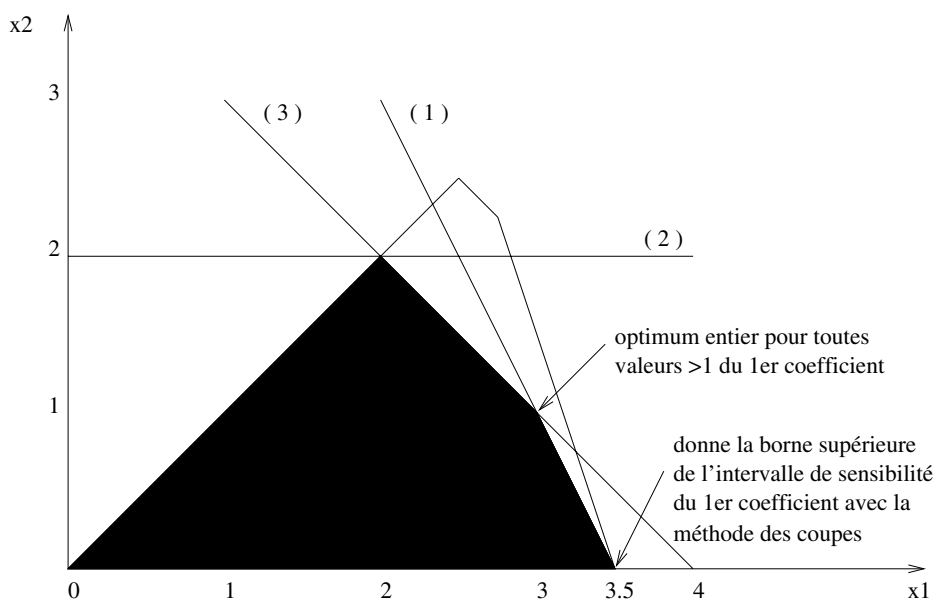

Figure 1. Exemple d'un problème en $0-1$ de Garfinkel et Nemhauser résolu par la méthode des coupes.

Le problème initial est résolu puisque la solution optimale par $(3,1)$ est à composantes entières (voir Fig. 1). Lorsque le calcul de l'intervalle de sensibilité découlant de la méthode de coupes est appliqué on trouve que la borne supérieure de l'intervalle de sensibilité associé à $c_{1}=2$ est bornée par la valeur de $c_{1}$ associée au point extrême adjacent $(3,5,0)$. Cette borne supérieure n'est pas la borne supérieure exacte de l'intervalle de sensibilité associé à $c_{1}$. En effet pour toute valeur $c_{1}>1$ la solution $x_{1}=3$ et $x_{2}=1$ reste optimale.

\subsubsection{Conditions basées sur la dualité}

Cette approche, due à Bell et Shapiro [2], consiste à faire l'analyse de sensibilité de la fonction objectif du problème $(P)$, en se basant sur les conditions 
d'optimalité issues de la théorie de la dualité convergente. Plus précisément, les auteurs considèrent le problème du sac à dos multidimensionnel, c'est-à-dire le problème $(P)$ dans lequel $X=\left\{x \in\{0,1\}^{n} \mid A_{1} x \leq b_{1}, \ldots, A_{m} x \leq b_{m}\right\}$, avec $c, A_{1}, . ., A_{m} \in \mathbb{N}^{n}$ et $b_{1}, . ., b_{m} \in \mathbb{N}$. Étant donné $u \in \mathbb{R}_{+}^{s}$, où $s \in\{1, . ., m-1\}$, on envisage la relaxation Lagrangienne de $(P)$ associée à la dualisation des $s$ premières contraintes :

$$
\begin{array}{lll}
(R L(u)) & \max & \sum_{1 \leq i \leq s} u_{i} b_{i}+\left(c-\sum_{1 \leq i \leq s} u_{i} A_{i}\right) x \\
\text { s.c } & x \in X_{R L}
\end{array}
$$

où $X_{R L}=\left\{x \in\{0,1\}^{n}, \mid A_{s+1} x \leq b_{s+1}, \ldots, A_{m} x \leq b_{m}\right\}$. Le dual Lagrangien est alors défini par

$$
\begin{array}{ll}
(D) \quad \min & v(R L(u)) \\
\text { s.c } & u \in \mathbb{R}_{+}^{s}
\end{array}
$$

et on sait que $v(D) \geq v(P)$.

La théorie de la dualité convergente est une méthode constructive [10,11,41] qui consiste à engendrer une séquence de problèmes convergeant vers un problème dual $(D)$ tel que $v(D)=v(P)$. Le calcul de l'intervalle de sensibilité proposé par Bell et Shapiro [2] porte sur un coefficient $c_{k}$ tel que $x_{k}^{*}=0$ (les auteurs ne traitent pas le cas $\left.x_{k}^{*}=1\right)$, où $x^{*}$ désigne la solution optimale de $(P)$. On sait qu'alors l'intervalle de sensibilité est donné par $I_{k}=\left[0, \bar{c}_{k}\right]$. Le calcul de $\bar{c}_{k}$ découle (Sect. 7.1.3) de l'inégalité

$$
v\left(R L^{\prime}\left(u^{*}\right) \mid x_{k}=1\right) \leq v\left(R L^{\prime}\left(u^{*}\right) \mid x_{k}=0\right)
$$

où $u^{*}$ et $\left(R L^{\prime}\right)$ désignent respectivement le multiplicateur optimal solution de $(D)$, le problème $(R L)$ dans lequel le coefficient $c_{k}$ est remplacé par $c_{k}^{\prime} \geq 0$.

On a alors d'une part, $v\left(R L^{\prime}\left(u^{*}\right) \mid x_{k}=0\right)=v(D)$, et d'autre part

$$
v\left(R L^{\prime}\left(u^{*}\right) \mid x_{k}=1\right)=\sum_{1 \leq i \leq s} u_{i}^{*} b_{i}+\left(c_{k}^{\prime}-\sum_{1 \leq i \leq s} u_{i}^{*} A_{i k}\right)+v(H)
$$

où $(H)$ est défini par

$$
\begin{aligned}
(H) \quad \max & \sum_{j \in\{1, . ., n\} \backslash\{k\}}\left(c_{j}-\sum_{1 \leq i \leq s} u_{i}^{*} A_{i j}\right) x_{j} \\
\text { s.c } & \sum_{j \in\{1, \ldots, n\} \backslash\{k\}} A_{i j} x_{j} \leq b_{i}-A_{i k} \quad i=s+1, . ., m \\
& x_{j} \in\{0,1\} \quad \forall j \in\{1, \ldots, n\} \backslash\{k\} .
\end{aligned}
$$

Ainsi $\bar{c}_{k}$ est obtenu à partir de la plus grande valeur de $c_{k}^{\prime}$ telle que $(*)$ est vraie, i.e. $I_{k}=\left[0, v(D)-v(H)-\sum_{1 \leq i \leq s} u_{i}^{*}\left(b_{i}-A_{i k}\right)\right]$. Cela nécessite bien sûr la résolution du problème $(H)$.

\subsection{RÉSOLUTION PAR RÉOPTIMISATION}

Cette section donne un aperçu des approches existantes en distinguant le cas de la réoptimisation dans une séquence de deux instances d'un même problème (Sect. 7.2.1), du cas où la séquence contient plus de deux instances (Sect. 7.2.2). 


\subsubsection{Séquence de deux instances d'un même problème}

On s'intéresse ici à la réoptimisation dans une séquence de deux instances d'un même problème linéaire en $0-1(P)$. C'est-à-dire qu'après résolution d'une instance de $(P)$, on veut chercher à résoudre efficacement une seconde instance de $(P)$.

Nous avons vu dans la section 7.1.3 comment il est possible d'exploiter l'arborescence obtenue en résolvant par exploration arborescente le problème $(P)$ initial afin de déterminer l'intervalle de sensibilité d'un coefficient en aval de cette résolution. On désire exploiter la résolution de $(P)$ pour déterminer la solution optimale d'un problème $\left(P^{\prime}\right)$ obtenu en modifiant les données de l'objectif du problème $(P)$ :

$$
\begin{array}{lll}
\left(P^{\prime}\right) & \max & c^{\prime} x \\
\text { s.c } & x \in X .
\end{array}
$$

La modification de l'objectif n'est plus ici limitée à une donnée unique. Tous les coefficients de $c^{\prime}$ peuvent être différents de ceux de $c$.

Les méthodes proposées dans la littérature [36-39] consistent à appliquer le principe décrit dans la section 7.1.3 pour un intervalle de sensibilité. On explicite encore les ensembles Ep et Ec (définis dans la Sect. 7.1.3) associés à l'arborescence de résolution de $(P)$. Compte tenu du fait que $(P)$ et $\left(P^{\prime}\right)$ ont le même domaine réalisable, il s'agit de réévaluer les solutions complètes (i.e. les solutions de Ec) et de poursuivre l'énumération à partir des solutions partielles (i.e. les solutions de Ep) puisque le test d'élagage qui était valide pour l'objectif de $(P)$ ne reste plus valide en général pour l'objectif de $\left(P^{\prime}\right)$. L'algorithme consiste alors à :

1. résoudre $(P)$ par branch \& bound en sauvegardant les solutions partielles Ep et complètes Ec engendrées;

2. réévaluer l'ensemble des nœuds pendants de l'arborescence construite en phase 1 avec l'actuel objectif $c^{\prime}$ de $\left(P^{\prime}\right)$. Continuer le branch \& bound à partir des solutions partielles de Ep qui ne violent plus le test délagage au regard de $c^{\prime} . v\left(P^{\prime}\right)$ sera égal à la valeur de la meilleure solution trouvée par ce processus.

Notons que cette technique de réoptimisation est applicable aux problèmes non linéaires.

\subsubsection{Séquence de plusieurs instances d'un même problème}

Supposons maintenant qu'on veuille résoudre par réoptimisation une séquence finie d'instances d'un même problème linéaire en variables 0-1. C'est un cadre d'étude qui a été peu abordé dans la littérature (voir $[9,35,49,49]$ ).

Historiquement c'est Robert Nauss qui le premier dans son Ph.D. [35] s'est vraiment penché sur cette question. Il considère une suite finie d'instances de sac à dos unidimensionnels connus a priori, et qui ne diffèrent que du second membre des contraintes. Pour résoudre chaque sac à dos, Nauss envisage alors d'utiliser la technique de réoptimisation qui consiste à exploiter l'arborescence d'un sac à dos résolu pour résoudre le suivant. Ses expériences numériques montrent qu'il est coûteux de réévaluer les solutions partielles et de développer une arborescence de 
branch \& bound pour une instance à partir de l'arborescence obtenue lors de la résolution précédente.

Au contraire du travail effectué par Robert Nauss, qui envisage un enchaînement d'instances sans aucune relation a priori, nous avons envisagé une séquence d'instances de sac à dos unidimensionnels obtenue par une méthode de sous-gradient, pour résoudre le dual Lagrangien du biknapsack. Nous avons employé dans $[47,49]$ des techniques de réoptimisation à l'intérieur des phases de prétraitement et d'exploration pour la résolution de chaque instance. Ces techniques portent sur le calcul de bornes supérieure et inférieure de la valeur de l'instance courante de sac à dos, ainsi que sur la résolution par une méthode de branch \& bound. Nous montrons comment il est possible d'exploiter des informations pertinentes obtenues à l'itération précédente, pour d'une part calculer en temps raisonnable des bornes de bonne qualité, d'autre part exploiter l'arborescence obtenue précédemment afin d'accélérer la résolution. Ces techniques de réoptimisation ont permis d'obtenir des gains de l'ordre de $70 \%$.

Dans la cadre de la résolution d'une séquence d'instances de plus court chemin avec fenêtre de temps obtenue par génération de colonnes, Desrochers et Soumis ont proposé dans [9] un algorithme de réoptimisation pour résoudre chaque plus court chemin. Un gain en temps de deux à dix fois a pu être mis en évidence dans ce travail.

\subsection{DÉtermination Du RAYON DE Stabilité}

La première expression analytique du rayon de stabilité, due à Leontev [30], est la suivante :

$$
\rho(c)=\min _{x^{\prime} \notin \Omega(P)} \max _{x \in \Omega(P)} \frac{\left|c x^{\prime}-c x\right|}{C+C^{\prime}-2 C C^{\prime}}
$$

où pour $x$ et $x^{\prime}$ dans $X$ données,

$$
\begin{aligned}
C & =\left|\left\{i=1, . ., n \mid x_{i}=1\right\}\right| \\
C^{\prime} & =\left|\left\{i=1, . ., n \mid x_{i}^{\prime}=1\right\}\right| \\
C C^{\prime} & =\left|\left\{i=1, . ., n \mid x_{i}^{\prime}=x_{i}\right\}\right| .
\end{aligned}
$$

Précisons que la notation $|$.$| a ici deux sens : \left|c x^{\prime}-c x\right|$ est une valeur absolue alors que $|\{.\}$.$| est la cardinalité d'un ensemble.$

Pour une utilisation pratique, il est nécessaire de chercher à obtenir une formulation simplifiée en exploitant la structure spécifique du problème à étudier [5].

Considérons comme illustration le problème spécifique de l'arbre couvrant de poids minimum d'un graphe simple $G=(V, E)$ où $V$ et $E$ sont respectivement l'ensemble des sommets et l'ensemble des arêtes. Étant donné le vecteur des poids $c \in \mathbb{R}^{n}$ ( $n$ étant le nombre d'arêtes), le problème de l'arbre couvrant de poids minimum

$$
\begin{array}{ll}
(P) \quad \min & c x \\
\text { s.c } & x \in X
\end{array}
$$


où $X=\{$ arbres couvrants de $G\}$, a été beaucoup étudié [46,51]. Gordeev a montré dans [15] que si $|\Omega(P)|=1$, c'est-à-dire que $(P)$ n'admet qu'une solution optimale $x^{*}$, la complexité de calcul du rayon de stabilité de $x^{*}$ est égale à celle de calcul de l'intervalle de sensibilité de $c_{k}$ tel que $x_{k}^{*}=1$. En effet la formulation analytique du rayon de stabilité

$$
\rho(c)=\min _{x \notin \Omega(P)} \frac{\left|c x-c x^{*}\right|}{C+C^{*}-2 C C^{*}}
$$

où

$$
\begin{aligned}
C & =\left|\left\{i=1, . ., n \mid x_{i}=1\right\}\right| \\
C^{*} & =\left|\left\{i=1, . ., n \mid x_{i}^{*}=1\right\}\right| \\
C C^{*} & =\left|\left\{i=1, . ., n \mid x_{i}^{*}=x_{i}\right\}\right|
\end{aligned}
$$

peut être simplifiée. Soit $\hat{X}$ l'ensemble des arbres couvrants possédant $|V|-2$ arêtes communes avec l'arbre couvrant optimal. Le dénominateur de $\rho(c)$ devient alors égal à 2. On montre ainsi dans [15] que

$$
\rho(c)=\min \left\{c x-c x^{*} \mid x \in \hat{X}\right\} / 2 .
$$

On peut encore reformuler cette expression en considérant pour tout élément $i \in$ $\{1, . ., n\}$ tel que $x_{i}^{*}=1$, un coût marginal $d_{i}$ défini par

$$
d_{i}=\min \left\{c_{j}-c_{i}\right\}
$$

où le minimum est pris sur l'ensemble des $j \in\{1, . ., n\} \backslash\{i\}$ tel que $x_{j}^{*}=0$ et tel que la solution $\bar{x}$ définie par :

$$
\bar{x}_{k}= \begin{cases}1 & k=j \\ 0 & k=i \\ x_{k}^{*} & k \neq i, j\end{cases}
$$

appartient à $\hat{X}$. Le coût marginal d'une arête de l'arbre couvrant optimal est donc le surcoût obtenu lorsqu'au lieu de prendre cette arête on en choisit une autre n'appartenant pas à l'arbre couvrant optimal.

On a alors d'après [15],

$$
\rho(c)=\min \left\{d_{i}, i \in\{1, . ., n\} \mid x_{i}^{*}=1\right\} / 2 .
$$

À partir de cette expression il est possible d'appliquer un algorithme simple décrit dans [15] pour déterminer le rayon de stabilité. Néanmoins Gordeev a proposé d'utiliser l'algorithme de Tarjan présenté dans [46] pour déterminer les intervalles de sensibilité individuels $\left[\underline{c}_{i}, \bar{c}_{i}\right]$, des poids $c_{i}$ lorsque $x_{i}^{*}=1$. À partir de l'expression du coût marginal, on obtient

$$
\rho(c)=\min \left\{c_{i}-\underline{c}_{i}, \bar{c}_{i}-c_{i}, x_{i}^{*}=1\right\} / 2 .
$$


Comme il s'agit d'un problème de minimisation, $x^{*}$ reste optimale si dans le graphe $G$ la valeur des $c_{j}$ est diminuée d'une quantité quelconque, et on a $\underline{c}_{i}=-\infty$ (borne évidente de l'intervalle de sensibilité). D'où

$$
\rho(c)=\min \left\{\bar{c}_{i}-c_{i}, x_{i}^{*}=1\right\} / 2 .
$$

Si pour chaque $c_{i}$ tel que $x_{i}^{*}=1, \bar{c}_{i}$ est connu, alors $\rho(c)$ peut-être déterminer en $O(|E|)$. Tarjan [46] a montré que l'ensemble des $\bar{c}_{i}$ peut-être calculé en $O(|E| a(|E|,|V|))$, avec $a(|E|,|V|)=\min \left\{i, \log ^{(i)}|V| \leq|E| /|V|\right\}$ où $\log ^{(i)}$ désigne $i$ compositions de $\log$. Ainsi $\rho(c)$ a une complexité en $O(|E| a(|E|,|V|))$ pour le problème de l'arbre couvrant de poids minimum.

Divers autres problèmes d'optimisation combinatoire ont fait l'objet d'études spécifiques du rayon de stabilité, notamment pour :

- le problème de Steiner (de nombreux algorithmes pour déterminer le rayon de stabilité pour ce problème sont basés sur les algorithmes pour les arbres couvrants de poids minimum $[15,46,51])$;

- le problème d'ordonnancement $[42,45]$;

- le problème de minimisation d'une forme booléenne linéaire [27, 43];

- le problème du voyageur de commerce [33].

\section{Conclusion et PerspeCtives De ReCherche EN ANAlyse DE SENSIBILITÉ}

Cette synthèse sur l'analyse de sensibilité dédiée aux problèmes linéaires en $0-1$, montre la diversité des travaux dévolus à cette thématique. Tout en tentant d'unifier les différentes formes d'analyse de sensibilité, un accent particulier a été mis sur l'analyse de sensibilité de la fonction objectif. Des travaux plus spécifiques sur l'analyse de sensibilité relative aux contraintes sont détaillés dans $[3,6,12,25$, 40].

Les directions de recherche actuelles sur l'étude de l'analyse de sensibilité portent essentiellement sur :

- l'exploitation des conditions d'optimalité dans le cadre des méthodes autres que le branch \& bound;

- la détermination du rayon de stabilité pour des problèmes spécifiques. La prise en compte de contraintes structurées aboutit à une simplification de l'analyse (comme nous l'avons illustré à travers le problème de l'arbre couvrant);

- le développement de techniques pour déterminer la région de stabilité des solutions $\epsilon$-approchées, compte tenu de la difficulté de l'analyse postoptimale. Étant donné une solution optimale et une solution $\epsilon$-approchée $x$, on s'intéresse à l'ensemble des variations des données telles que $x$ reste une solution $\epsilon$-approchée. Citons [27, 43, 45]; 
- l'étude de la réoptimisation répétitive qui tire profit de la connexité des problèmes sous-jacents à certains algorithmes itératifs. Il est alors possible d'exploiter les résultats sur la réoptimisation d'un problème à un autre comme nous l'avons vu lorsqu'un problème linéaire est résolu par branch \& bound. Mais il est souvent nécessaire d'avoir une vision globale du comportement de l'algorithme itératif pour construire des techniques de réoptimisation efficaces $[9,35,47,48]$.

\section{RÉFÉRENCES}

[1] E. Balas, An additive algorithm for solving linear programs with zero-one variables. Oper. Res. 13 (1965) 517-546.

[2] D.E Bell and J.F Shapiro, A convergent duality theory for integer programming. Oper. Res. 1 (1977) 467-477.

[3] C. Blair, Sensitivity analysis for knapsack problems : a negative result. Discrete Appl. Math. 81 (1998) 133-139.

[4] P.J. Carstensen, Complexity of some parametric integer and network programming problems. Math. Program. 26 (1983) 64-75.

[5] N. Chakravarti and A.P.M. Wagelmans, Calculation of stability radius for combinatorial optimization problems. Oper. Res. Lett. 23 (1999) 1-7.

[6] W. Cook, A.M.H. Gerards, A. Schrijver and E. Tardos, Sensitivity theorems in integer linear programming. Math. Program. 34 (1986) 251-264.

[7] A. Crema, An algorithm for the multiparametric 0-1 integer linear programming problem relative to the objective function. Eur. J. Oper. Res. 125 (2000) 18-24.

[8] A. Crema, The multiparametric 0-1 integer linear programming problem : A unified approach. Eur. J. Oper. Res. 139 (2002) 511-520.

[9] M. Desrochers and F. Soumis, A reoptimization algorithm for the shortest path problem with time windows. Eur. J. Oper. Res. 35 (1988) 242-254.

[10] M.L. Fisher, W.D. Northup and J.F. Shapiro, Using duality to solve discrete optimization problems : Theory and computational experience. Math. Prog. Study 3 (1975) 56-94.

[11] M.L. Fisher and J.F. Shapiro, Constructive duality in integer programming. SIAM J. Appl. Math. 27 (1974) 31-52

[12] T. Gal and H.J. Greenberg, Advances in sensitivity analysis and parametric programming. Kluwer Academic Publishers, Boston-Dordrecht-London (1997).

[13] R.S Garfinkel and G.L. Nemhauser, Integer programming. Wiley, New York (1972).

[14] A.M. Geoffrion and K. Nauss, Parametric and postoptimality analysis in integer linear programming. Manage. Sci. 23 (1977) 453-466.

[15] E.N. Gordeev, The complexity of stability study in discrete optimization problems. Cybernetics questions 133, computer center of the USSR academy of sciences, Moscow (1989) 41-77 (en russe).

[16] E.N. Gordeev, Solution stability in a shortest path problem. Discrete Math. 1 (1989) 45-56 (en russe).

[17] H.J. Greenberg, An annoted bibliography for post-solution analysis in mixed integer programming and combinatorial optimization, in Advances in Computational and Stochastic Optimization, Logic Programming and Heuristic Search, edited by D.L. Woodruff. Kluwer Academic Publishers, Boston, MA (1998) http ://carbon.cudenver.edu/ hgreenbe/aboutme/pubrec.html

[18] D. Gusfield, Sensitivity analysis for combinatorial optimization. Memo. No. UCB/ERL M80/22, Electronics Research Laboratory, Univ. of California, Berkeley, California (1980).

[19] D. Gusfield, Parametric combinatorial computing and a problem of program module distribution. J. Association for Computing Machinery 30 (1983) 551-563. 
[20] S.V. Hoesel and A. Wagelmans, Sensitivity analysis of the economic lot-sizing problem. Discrete Appl. Math. 45 (1993) 291-312.

[21] S.V. Hoesel and A. Wagelmans, On the complexity of postoptimality analysis of $0 / 1$ programs. Discrete Appl. Math. 91 (1999) 251-263.

[22] S. Holm and D. Klein, Three methods for postoptimal analysis in integer linear programming. Math. Prog. Study 21 (1984) 97-109.

[23] L. Jenkins, Parametric-objective integer programming using knapsack facets and Gomory cutting planes. Eur. J. Oper. Res. 31 (1987) 102-109.

[24] L. Jenkins, Parametric methods in integer linear programming. Ann. Oper. Res. 27 (1990) 77-96.

[25] D. Klein and S. Holm, Discrete right hand-side parametrization for linear integer programs. Eur. J. Oper. Res. 2 (1978) 50-53.

[26] D. Klein and S. Holm, Integer programming postoptimal analysis with cutting planes. Manage. Sci. 25 (1979) 64-72.

[27] M.Y. Kovalyev and Y.N Sotskov, $\epsilon$-approximate solution stability of boolean linear form minimization. Vesti Akad. Navuk BSSR, Ser. Fiz.-Mat. Navuk 22 (1990) 111-116 1990 (en russe).

[28] V.K. Leontev, Stability in traveling salesman problem. At. i Mat. Fiz. 15 (1975) 1298-1309 (en russe).

[29] V.K. Leontev, Stability in combinatorial choice problems. Dokl. Akad. Nauk SSSR 1 (1976) 23-25 (en russe).

[30] V.K. Leontev, Stability in linear discrete problems. Cybernet. Probl. 35 (1979) 169-184 (en russe).

[31] M. Libura, Sensitivity analysis for integer knapsack problem. Archiwum Automatyki i Telemechanik 22 (1977) 313-322 (en polonais).

[32] M. Libura, Optimality conditions and sensitivity analysis for combinatorial optimization problems. Control Cybernet. 25 (1996) 1165-1180.

[33] M. Libura, E.S. Van der Poort, G. Sierksma and J.A.A. Van der Veen, Stability aspects of the traveling salesman problem based on $k$-best solutions. Discrete Appl. Math. 87 (1998) 159-185.

[34] E. Loukakis and A.P. Muhlemann, Parametrisation algorithms for the integer linear programs in binary variables. Eur. J. Oper. Res. 17 (1984) 104-115.

[35] K. Nauss, Parametric integer programming. Ph.D. thesis, Western Management Science Institute, UCLA (1975).

[36] C.J. Piper and A. Zoltners, Implicit enumeration based algorithms for post-optimising zeroone programs. Naval Res. Logist. Quarterly 22 (1975) 791-809.

[37] C.J. Piper and A. Zoltners, Some easy postoptimality analysis for zero-one programming. Manage. Sci. 22 (1976) 759-765.

[38] G.M. Roodman, Postoptimality analysis in zero-one programming by implicit enumeration. Naval Res. Logist. Quarterly 19 (1972) 435-447.

[39] G.M. Roodman, Postoptimality analysis in zero-one programming by implicit enumeration. The mixed integer case. Naval Res. Logist. Quarterly 21 (1974) 595-607.

[40] L. Schrage and L. Wolsey, Sensitivity analysis for branch and bound integer programming. Oper. Res. 33 (1985) 1008-1023.

[41] J.F. Shapiro, Generalized lagrange multipliers in integer programming. Oper. Res. 19 (1971) 68-76.

[42] Y.N. Sotskov, Stability of an optimal schedule. Eur. J. Oper. Res. 55 (1991) 91-102.

[43] Y.N Sotskov, The stability of the appoximate boolean minimization of a linear form. USSR Comput. Math. Math. Phys. 33 (1993) 699-707.

[44] Y.N. Sotskov, V.K. Leontev and E.N. Gordeev, Some concepts of stability analysis in combinatorial optimization. Discrete Appl. Math. 58 (1995) 169-190.

[45] Y.N Sotskov, A.P.M. Wagelmans and F. Werner, On the calculation of the stability radius of an optimal or an approximate schedule. Ann. Oper. Res. 83 (1998) 213-252. 
[46] R.E. Tarjan, Sensitivity analysis of minimum spanning trees and shortest path trees. Inform. Proc. Lett. 14 (1982) 30-33.

[47] B. Thiongane, Réoptimisation dans le dual lagrangien du biknapsack en variables 0-1. Thèse de Doctorat en Informatique, Université de Paris 13 (2003).

[48] B. Thiongane, A. Nagih and G. Plateau, Adapted step size in a 0-1 biknapsack lagrangean dual solving algorithm. En révision pour Ann. Oper. Res. (2002).

[49] B. Thiongane, A. Nagih, and G. Plateau, Lagrangean heuristics combined with reoptimization for the 0-1 biknapsack problem. En révision pour Discrete Appl. Math. (2002).

[50] A.P.M Wagelmans, Sensitivity analysis in combinatorial optimization. Ph.D. thesis, Eramsus University, Rotterdam (1990).

[51] P. Winter, Steiner problem in networks: A survey. Networks 17 (1987) 129-167.

To access this journal online: www.edpsciences.org 